\title{
SIENA: Grid and Cloud Standards for e-Science and beyond
}

\author{
Silvana Muscella, Nick Ferguson ${ }^{1}$, Stephanie Parker \\ OGF.eeig, Italy \\ E-mail: \{n.ferguson, s.muscella, s.parker\}@trust-itservices.com
}

Rosa M. Badia, Daniele Lezzi

BSC, Spain

E-mail:\{daniele.lezzi, rosa.m.badia\}@bsc.es

David Wallom ${ }^{2}$

Oxford e-Research Centre, UK

E-mail: david.wollom@oerc.ox.ac.uk

\section{Mirco Mazzucato, Elisabetta Ronchieri}

INFN, Italy

E-mail: elisabetta.ronchieri@cnaf.infn.it, mirco.mazzucato@pd.infn.it

James Ahtes

ATOS, Spain

E-mail: james.ahtes@atosresearch.it

\section{Johannes Watzl}

LMU, Germany

E-mail: watzl@nm.ifi.lmu.de

\section{Martin Antony Walker}

Independent Consultant, France

E-mail: martin . antony.walker@gmail.com

\section{John Borras}

OASIS, UK

E-mail: johnaborras@yahoo.co.uk

\section{Carlo Daffara}

Conecta, Italy

E-mail: cdaffara@conecta.it

\footnotetext{
${ }^{1}$ Abstract editor, Corresponding author.

${ }^{2}$ Speaker.
} 
The adoption of open standards-based interoperable grid and cloud computing infrastructure is a way to support research in Europe that can be empowered through ubiquitous, trusted, and easy trans-national access to services for data, computation, communication and collaborative work. SIENA is the European Commission-funded project whose roadmap assesses the situation, identifies issues and makes recommendations on the adoption and evolution of grid and cloud standards for e-science and beyond. These recommendations look to continue the dialogue between public sector officials, industry and Standard Development Organizations over the deployment of cloud based services for the scientific community and public procurement.

This paper presents the results of the SIENA roadmap together with its recommendations that are focused on the work of Europe's Distributed Computing Infrastructure initiatives in advancing the standards landscape, especially with regard to the adoption of cloud standards such as OCCI, CDM and OVF.

EGI Community Forum 2012 / EMI Second Technical Conference, Munich, Germany

26-30 March, 2012 


\section{Introduction}

SIENA [1], Standards and Interoperability for eInfrastructure implemeNtation initiAtive, was funded by European Commission (EC) under the 7th Framework Programme (FP7) and ran from June 2010 - June 2012.

The project was the first initiative to bring to the same table standardization bodies to support the analysis of open standards-based interoperable grid and cloud computing infrastructures. The Standard Development Organizations (SDOs) that have contributed include: the Open Grid Forum (OGF), the Institute for Electrical and Electronics Engineers Standards Association (IEEE SA), the European Telecommunications Standards Institute (ETSI), the Distributed Management Task Force (DMTF), the Organization for the Adoption of Structured Information Standards (OASIS), the Storage Networking Industry Association (SNIA), International Telecommunication Union Telecommunication Standardization Sector (ITU-T) and World Wide Web Consortium (WC3).

The primary focus of the SIENA initiative was European electronic infrastructure for research which takes into consideration the points of view of both the people responsible for infrastructure creation and operation, such as service and application developers, and the "end users", for example, researchers in science, the arts and humanities. The focus on standards and interoperability and the offer of services through European grid and cloud infrastructures will benefit the education sector, and will also be relevant for e-government and commercial enterprises that can take advantage of the substantial research background that underlies most einfrastructures.

The main output of the project was the SIENA Roadmap on Distributed Computing Infrastructure for e-Science and Beyond in Europe [2]. With a foreword by Neelie Kroes, Vice President of the European Commission, the Roadmap assesses the current situation and challenges regarding the adoption and evolution of open standards-based interoperable grid and cloud computing infrastructure to support research in Europe. The document also sets out a clear set of Calls for Action necessary for this to become a reality.

SIENA has also created a critical mass of community engagement; a requirement to fulfill its wide roles as a facilitator of collaboration and cooperation among Europe's distributed computing landscape stakeholders through a series of dissemination and outreach communities.

The self-sustaining Cloudscape [3] series of events has found its place as a key cloud computing event in Europe, because of the direct participation of the very same stakeholders detailed in this report, as well as SIENA's leading connections in the standards development community. Cloudscape V [3], 27-28 February 2013, Brussels, will see a continuance of discussion on the SIENA Roadmap calls to action with updates on the progress of each point and the recommendations serving as a key part of discussion and debate.

Finally, SIENA has been instrumental in supporting the drive for the adoption of open standards by synergising with key SDOs to establish Cloud plug fest which sees the collaboration of major SDOs. SIENA has also supported DCI initiatives and has played an integral bridging role between EGI, DCI projects and SDOs for the EGI Federated Virtualisation Task Force. 
International collaboration has also been forged through the lifetime of the project, in particular with the National Institute of Standards and Technology (NIST), which lead to the mapping of NIST Use Cases and Cloud Usage Scenarios with European e-Science developments. SIENA was cited in the NIST Cloud Computing Standards Roadmap.

\section{Challenges in the adoption of cloud computing}

Powerful economic and environmental forces are driving a major evolution in the way computing resources are provisioned for user communities in research, in government and the public sector, and in industry. Economies of scale are driving consolidation of such resources into a smaller number of ever larger data centers ${ }^{3}$. Considerations of the cost of powering and cooling huge concentrations of electronic equipment, together with environmental concerns, drive the placing of such data centers in geographic locations where power is plentiful and inexpensive.

These forces and their consequences both enable and drive the move towards a utility computing model. The latest manifestation of this model is cloud computing. The dynamic flexibility and reduced cost of accessing resources in the cloud are beginning to overwhelm most other considerations on provisioning IT resources and providing services to many user communities ${ }^{4}$. As suggested by analysts such as Gartner, cloud computing represents a service provider-consumer model and is driving a fundamental shift in the consumption model of IT functionality.

Such a fundamental shift from asset ownership to service provisioning poses numerous challenges to all players in the transition.

Many of the general challenges ${ }^{5}$ to the adoption of cloud computing are shared by cloudbased e-infrastructures for research. These include overall coordination of projects and programmes, requirements identification, several issues associated with standardization, security, education, legal impediments, and globalization.

- Overall coordination of projects and programmes. A vast array of projects and programmes devoted to cloud computing exists, with new ones appearing almost daily [4]. The EC has a large collection of white papers and communications relevant to cloud computing, some of which are currently open for public consultation [5, 6] and comment, and can therefore be expected to change in the near term. There is so much going on that an active on-going openly accessible "cloud watch" would be very helpful.

- Conflicting and inappropriate requirements. The identification and prioritization of cloud computing requirements is an essential part of establishing security, portability and interoperability in cloud computing through relevant standards. A danger in this process is that conflicting or inappropriate requirements be imposed. For example, a requirement that a customer be able to specify the geographical location of data would be fatal for

\footnotetext{
${ }^{3}$ Data centers with hundreds of thousands of computational and storage units are not uncommon. A recently announced data center near Dallas, Texas will have 3,600 square meters of raised floor under one roof.

${ }^{4}$ Some important applications will continue to require dedicated high-performance computers.

${ }^{5}$ Chiemi Hayashi: presentation at the OASIS International Cloud Symposium, op cit.
} 
small- and medium-sized businesses proposing cloud services in Europe due to the high cost of reliable storage services ${ }^{6}$.

- Standardization challenges. As in the case of projects and programmes, the cloud computing standardization landscape is complicated and confusing with many different organizations and interests involved in cloud standardization efforts [7]. The US National Institute of Standards and Technology (NIST) [8] has compiled an inventory [9] of standards relevant to cloud computing, and has identified a core suite of emerging highlevel cloud-specific standards ${ }^{7}$, although, and as the NIST document points out, standards from other domains such as web services, the internet, and grid computing are available, and can support the functions and requirements of cloud computing.

- Security challenges [10]. Industry and government see risk in completely different ways ${ }^{8}$ (accept and manage, balance costs, clear lines of responsibility vs. eliminate risk, zero tolerance, ill-defined lines of responsibility). The vast majority of government data has nothing to do with national security ${ }^{9}$. In most cases for both industry and government, geographical location of data should not be relevant ${ }^{10}$. It has been argued that with appropriate standards in place, moving from proprietary in-house computing to cloud computing improves security ${ }^{11}$.

- Data Management challenges. There are a number of significant data management issues relating to the vast amount of data collected and used by research projects. These include ownership and governance of the data, creating trust in the data, sharing and reuse of the data and the long term preservation of the data. All of these issues are addressed in the recent report from the High Level Expert Group on Scientific Data [11]. We support the recommendations of this report, and urge immediate attention to their implementation.

- Education challenge. Education of decision takers at all levels of business and government, including politicians, is needed to overcome social and cultural obstacles to cloud computing adoption, to overcome inertia, and to ensure that the standardization and security challenges are met appropriately.

- Challenges in understanding terminology and approaches. The availability of ever more capable electronic components (e.g. for processing, memory, and networks) at ever

\footnotetext{
${ }^{6}$ Reliable data access would require multiple storage facilities (for redundancy) in each country in which the service is offered, making the service prohibitively expensive. Such requirements have been voiced, e.g., http://ur1.ca/9f2sm.

These three standards, OVF (DMTF), OCCI (OGF), and CDMI (SNIA), are described in the first iteration http://goo.gl/ZcF8k of the SIENA roadmap (March 2011). See also the presentation on cloud computing standards work at NIST by Annie Sokol at http://goo.gl/XhcxH, especially slides 17-19.

${ }^{8}$ Scott Algeier, OASIS International Cloud Symposium, op cit.

${ }^{9}$ Ian McCormack, OASIS International Cloud Symposium, op cit.

${ }^{10}$ Megan Richards, in response to a question at the OASIS International Cloud Symposium (op cit.), pointed out that the oft-quoted US Patriot Act is very detailed and restricted in scope, and is "not a fishing license". Tim Cowen pointed out in his presentation on legal impediments to cloud computing that European law enforcement agencies are also able to obtain access to data, and not necessarily under conditions as strict as in the US. Cloudscape IV, 23-24 February 2012, Brussels, highlighted that the focus on the US Patriot Act often eclipses the larger issue. It is the divergence between European and US data that needs addressing to ensure both parts are compatible and aligned with trade agreements for the open market, http://ur1.ca/9f2v0.

${ }^{11}$ Marnix Dekker, OASIS International Cloud Symposium, op cit.
} 
lower prices leads to distortion of traditional terminology. The term "high performance computing” traditionally referred to use of systems in the Top 500 and related complex application enabling activities (e.g. parallel programming, achieving application scalability, etc.). In this strict sense, neither a grid nor a cloud is a supercomputer (although a supercomputer can be a node in a grid, or - in principle - be offered as a service in a cloud). Nowadays, one hears about high performance computing (HPC) in the cloud and HPC on the desktop, contributing to confusion for both consumers and providers.

- Legal impediments. The current fragmented and inconsistent legal and regulatory environment in Europe is seen as a major inhibitor to the deployment of cloud services. This is a potential problem for sustainability of Europe's DCIs through uptake of their assets. A dramatic example is the mime cast story [12] in which a rapidly growing UK firm with a cloud-based email service was obliged to seek further growth in North America because of the impracticality of providing the service in Europe due to uncertainties over cross-border data movement. A recent report [13] from the EC following their public consultation on the subject also highlights the many issues. Annex 5 [14] contains a brief on legal issues in cloud computing adoption.

- Globalization challenges. Several major governmental cloud computing programs are underway [15]. These are being pursued in the absence of an adequate set of agreed and accepted open standards that could guarantee security, portability, and interoperability of cloud services, yet the globalization of business implies that inter-cloud portability, cloud interoperability and cloud federation are global issues ${ }^{12}$.

- Entrepreneurship challenge. Encouragement and support for the creation of new cloudbased businesses could be a motor for wealth creation and employment for young people prepared to take risks and work hard. The knowledge, technical expertise and assets offered by the DCIs can be a basis for the creation of new products and services, either directly through the sustainability efforts described in chapter 5 and Annex 3 [14] or through new start-ups. Creation of an atmosphere conducive to such entrepreneurial activity is still a major challenge for Europe, in spite of many coordinated efforts designed towards product-oriented research like Eureka/EuroStars [16].

- Rapid Pace of Change. In cloud computing, the pace of change necessitates agility and flexibility. Efforts have to be made in Europe to support start-ups and facilitate acceleration of progress through appropriate legal, procurement and financial environment.

- Collaboration efforts. The open source communities and their open innovation process are paving the way to effective construction of new assets. Today these models and processes are significantly reused by industry and academic players. A significant support and legal framework needs to be provided for such initiatives to be sustainable and even more productive. Cloud computing is a very complex domain where isolated players will not be able to master the whole complexity. For these reasons, now is the time to take

${ }^{12}$ See, e.g., the Cloudscape IV Executive Summary (Takeaways), highlighting the significant experience in federation gained by European e-infrastructure communities. This experience could be shared with colleagues from NIST, the US National Science Foundation and around the globe (op cit.). 
advantage of the valuable European open source communities to develop common value that can form foundations for new and innovative businesses and services.

Europe is well positioned to address many of the enumerated challenges, and indeed to take a world leading position in cloud computing, provided key challenges are met, notably to unlock $^{13}$ and encourage a European cloud computing market for start-ups and small- and medium-sized enterprises. The EC Framework Programme on e-infrastructure [17] and research infrastructures [18] plays to key European competencies in research. The EGI [19] is the largest, most powerful, and most comprehensive distributed computing e-infrastructure supporting research in the world ${ }^{14}$. Europe can also capitalize on the achievements of DCIs that have placed special emphasis on cloud computing and made concerted efforts to share experiences in the implementation of standards. VENUS-C is among the European DCIs leading the way in usercentric approaches to the cloud, demonstrating its feasibility for the 'long tail of science' and supporting a number of small businesses. Two of its pilots are closely associated with EDGI, helping to bring its desktop grid infrastructure into the cloud and thus extend and integrate the e-infrastructure. As the work of the StratusLab project focuses on the IaaS layer, there has been strong support of standards development in this area. StratusLab has established interaction with OGF, following closely the work on OCCI as well as on network management standards. Although not strictly a standards-related group, StratusLab has worked with the HEPiX community to define an Appliance Marketplace metadata format and develop the respective service. Further, StratusLab and VENUS-C have sought to ensure productive collaboration by sharing experiences on implementations and use of OpenNebula, as well as clarifying interactions between IaaS and PaaS cloud infrastructures and relevant standards [20].

OpenNebula [21] is an open source project developing solutions for building and managing virtualized data centers and cloud infrastructures. The e-infrastructure users of OpenNebula include EGI, StratusLab, EDGI, and VENUS-C [22].

CompatibleOne [23] is an open source collaborative project providing a model and platform for the description and federation of heterogeneous cloud services.

The EGI-InSPIRE project [24], in collaboration with other EC-funded distributed computing initiatives and stakeholders ${ }^{15}$, is driving the transition through virtualization from a grid-based to a cloud-based e-infrastructure. In particular, the Federated Clouds Task Force [25] is working on the required standards and methodologies to implement a transition from grid- to cloud- based services for e-infrastructure provision to researchers in the European Research Area by building on current work. This task force has identified requirements from einfrastructure stakeholders (resource centers offering cloud services, technology providers tendering solutions, and user communities consuming cloud services) for eight key federated cloud capabilities. An ambitious standards and profile development process for these six key capabilities within the OGF [26] is planned, which will deliver recommendations for open

\footnotetext{
${ }^{13}$ Justin Pirie, mimecast cloud strategist: "Doing business in Europe is frankly a nightmare.”

${ }^{14}$ The EGI comprises 346 contributing resource centers in 57 countries, with four non-European operations centers. The infrastructure supports 13,800 users in 219 virtual organizations, running 28 million computational jobs per month on 338 thousand processors, backed up by 107 petabytes of disk and 113 petabytes of tape storage. The infrastructure delivers 75 million processor wall-clock hours of computation per month to its user communities.

${ }^{15}$ European Desktop Grid Initiative (EDGI), European Middleware Initiative (EMI), Initiative for Globus Europe (IGE), StratusLab, and VENUS-C (Virtual Multidisiciplinary Environments Using Cloud Infrastructure).
} 
standards by the end of 2012 through the DCI Federation Working Group (DCIFed-WG) [27]. Interaction with other SDOs with projects in this domain [28] will benefit the further development of appropriate standards leading to interoperability of future cloud computing infrastructures.

Imminent future efforts such as the EC-funded two-year pilot project Helix Nebula ${ }^{16}$ will explore a European Cloud infrastructure for science with a commercial basis. The project will map demand from the European Space Agency (ESA) and CERN and other large capacity use cases with IT integrators and telecom companies on the supply side. A pilot testing phase will precede a 2014 rollout. Partners in the Helix Nebula project include OpenNebula and EGI.eu in addition to thirteen European industrial partners.

\section{Call for Actions}

The following recommendations constitute a "Call for Action" on all stakeholders involved in the development of European e-infrastructure. These recommendations have been formulated and divided into immediate short term priority actions, and actions with a mediumterm timeframe. By short-term we mean within a maximum of twelve months, and by mediumterm we mean a maximum of three years. These timeframes are the maximum amount of time that we feel it should take to complete each call for action, although we anticipate that each could be completed well within that timeframe. They take into account the life expectancies of the current DCI projects, and the probability of new calls for new projects by the European Commission.

Within this overall plan there is a role for governments where the trend towards procurement of commercial cloud services by the public sector (including research) will generate interest in standards. There is also an important role for industry to play in the international standards dialog, implementation and certification processes, as well as in continued investments aimed at boosting European innovation.

\subsection{Immediate priority actions}

We offer a list of actions, building on work already begun across Europe and complementing efforts globally, which merit attention within the next twelve months:

\section{Determine optimum deployments of cloud computing for research}

An urgent initiative is required to review current deployments of cloud computing for eresearch and the broader scientific user and infrastructure communities at various service levels, (infrastructure-, platform- , software- , science- as-a-service) using a representative range of applications and determine the optimum deployment(s) for future use.

This will require the collection of case studies and careful requirements analysis, and interaction with infrastructure, standards, software and application stakeholders. The latter would help to understand where emphasis should be placed, such as at which cloud service

\footnotetext{
${ }^{16}$ See, e.g. Maryline Lengert, Helix Nebula - The Science Cloud, Position Paper, Cloudscape IV (op cit.) and presentation Helix Nebula - The Science Cloud. A Strategic Plan for a European Scientific Cloud Computing Infrastructure, Cloudscape IV (op cit.).
} 
level ${ }^{17}$ a particular application is focused. Taking into account the impact of ICT on the environment and the use of green technology, the criteria must not only be about pure performance but on overall efficiency as well.

\section{Strengthen, collaborative international dialogue for achieving interoperability and portability}

It is imperative that the current standardization dialog be strengthened and widened to involve all stakeholders, including SDOs, cloud collaborative working groups, open source and commercial infrastructure providers, and other global and regional public sector institutions, in order to guarantee adequate representation of all the needs and to leverage the inherent strengths of each group to the benefit of the cloud computing environment as a whole in the international arena. Continuous dialog with all key stakeholders internationally, on priority action plans is a foundation upon which to strengthen respective use cases, best practices and achieving true interoperability and portability. To this end, the EC and European member state governments should include direct support for funded activities to participate in relevant international activities. Furthermore, common 'synchronized' calls for proposals between the EC and the US National Science Foundation (NSF) and other national funding organizations, could strengthen international collaborations.

\section{Strive for a common approach for contributing to the European Digital Market}

A common approach for European Member States public administrations is necessary to help citizens and businesses profit fully from the European Union's digital single market. The eresearch communities' contribution to this goal should be through the EC putting in place plans for the long-term sustainability and reuse of the assets from existing DCI projects. A condition of approval for future DCI projects should be the creation of reusable assets wherever possible for potential uptake by European government, industry and e-research and more widely wherever feasible. As part of plans for sustainability and asset reuse, attention needs to be paid to avoiding multiple independent parallel developments offering almost identical functionalities, and to recognize that these developments can also span diverse areas such as transport, energy or health.

\section{Expand support for DCI efforts to provide mechanisms to federate across multiple cloud suppliers}

A major near-term initiative, supported by the EC, is required to enable federation across multiple globally located cloud providers, including those in the SME bracket, to support European participation in global research enterprises. This federation must build upon the participation of European communities to build the necessary standards, and implement the software profiles and a common administrative framework. The EGI project will be exploring such participation during 2012 since there is a need for a compendium of virtual machines (platforms) to be available for user communities to deploy and link together on European einfrastructures.

\footnotetext{
${ }^{17}$ Infrastructure, platform, or software.
} 
Introduce measures to provide open access to all relevant SDO standard documentation

The SDOs that can be regarded as producing open standards should be actively encouraged by the EC to share information and documentation about their standards work to avoid duplication of effort and provide better understanding to the many communities seeking to use their standards. Open standards that are readily available to whoever wishes to use them are necessary to support the procurement process by reducing the risk of becoming dependent on a single vendor. Vendors will implement standards if they are listed as technical specifications in calls for tender by public sector customers.

One way of achieving this action would be to build on the current plans of the EC's ADMS [29] project on sharing repository metadata which are very relevant to this need.

\section{Introduce business models for use of clouds by research}

A set of common business models for the use of cloud computing by research communities is required. In order for cloud computing to be used effectively for research, certain special cloud services and properties will be required which are not driven by egovernment or business applications. In some cases no good solution may be available, e.g. long-term data curation. There is general agreement that much research data must reside in the cloud, but there is currently no business model to support long-term sustainability, and government funding alone is not expected to be sufficient.

\section{Review public sector procurement regulations}

It is very important to allow the DCIs to liaise with governments to support large researchoptimized "private" clouds. To this end a close examination of the existing European and national public sector procurement regulations is necessary to identify and remove any barriers to the procurement and re-use of DCI assets by governments and other public sector organizations before these projects close. Aspects that need to be addressed include: should there be an open tender process to purchase any of the assets; could an overarching framework contract be put in place to handle the disposal of assets; does the OJEU [30] limit need to be changed; will the increasing trend for public sector procurements demanding solutions that use open standards be an obstacle for those DCI projects that have used proprietary or non- standard approaches.

\section{Re-use tangible and intangible assets produced by DCIs}

The DCIs should produce tangible and intangible assets together with associated information packages that can be made available to other parts of the public sector, SMEs and industry. The urgency of this action is necessary to protect the assets of those DCI projects that are scheduled to terminate in the short- to medium-term. The awareness of such information packages should be supported not only by DCI projects themselves, but also by the respective units of the funding organizations who should promote re-use of results in projects of other units where such results may be needed. As part of such packages, the DCIs should evaluate using a transparent procedure, explain the maturity of their offerings and include their sustainability plan for each asset. This will assist potential customers in making possible procurement and 
reuse/participation decisions. The production of this material should be a pre-requisite of future projects approved and funded by the EC.

This action should include necessary publically published reviews of the deliverables from the current DCIs such that confidence is raised in the products that have been generated.

\section{Establish an e-infrastructure Implementation Advisory Group}

An e-infrastructure Implementation Advisory Group (eIIAG) should be established under the auspices of the EC to provide communication across all relevant stakeholders, i.e. the einfrastructure community, the SDOs producing relevant standards, and those organizations and projects consuming their standards. The main roles of this group are to maintain a "cloud watch" on cloud computing projects, programs and service offerings; agree requirements and the standardization route for those requirements; share policy, regulatory and implementation advice; develop standards' profiles; promote interoperability testing and plug fests/tests; and establish a compliance testing regime, all of which are essential components to achieving true interoperability. Finally, the Group should maintain close liaison with the e-Infrastructure Reflection Group, eIRG [31].

EC-funded projects should be required to take recommendations of the Group into account in their work. The Group must have representation from major e-infrastructures like EGI and Partnership for Advanced Computing in Europe (PRACE) [32] in order to provide a single point of contact for e-infrastructure across Europe. To ensure that research communities have faith in its continued sustainability the Group must also have longer term funding (more than five years and possibly more than ten).

\subsection{Medium term priority actions}

Within a medium term timeframe the following actions merit attention:

\section{Co-ordinate global regulations for transnational data flows}

An action that allows Europe to take the lead in coordinating the harmonization of regulations on the flows of data across borders is required. Many governments place restrictions on allowing data to be held outside their jurisdictions, primarily because of concerns about the adequacy of data protection legislation in other countries. The longer this situation persists, the slower the take up of cloud computing by the public sector will be. Therefore, this problem needs to be rectified.

\section{Create an inventory of grid and cloud standards for research}

A shared inventory of grid and cloud standards should be developed and maintained for the e-research stakeholder community covering both domain specific and e-infrastructure capabilities, with pointers to proven interoperable implementations and warnings of defunct or unused standards. This inventory should result from an EC-commissioned independent review and validation of current and proposed open standards and be aligned with the NIST Cloud Standards Inventory ${ }^{18}$ and the outputs of other relevant grid and cloud collaboration groups.

The definition of a perhaps roughly defined but guiding reference model and associated standard-based reference architecture(s) should be explored as part of creating the inventory, in

\footnotetext{
${ }^{18}$ See NIST Inventory, op cit.
} 
order to provide a frame of reference for standard adoption in a wide area of potentially different ICT sectors.

\section{Conclusion}

"The SIENA Roadmap demonstrates that Europe has a huge potential for innovation. I encourage you all to pay particular attention to this Roadmap, to discuss it, to question it, bring it together with other aspects that may also need particular attention. Essentially to help calibrate it and bring its recommendations into actions so that Europe can be not only cloud friendly but also cloud active" Mario Campolargo, Director DG INFSO Emerging Technologies and Infrastructures at the EC, Cloudscape IV keynote on cloud computing for the Digital Agenda.

This paper has offered an overview of the SIENA roadmap and has paid particular attention to the challenges to the adoption of cloud computing. The paper has also focused on the set of broad calls for action which target all stakeholders involved in the development of European e-infrastructure. It is imperative that these recommendations are noted by interested parties and that action is taken.

The release of the second iteration of the SIENA Roadmap was timed to coincide with Cloudscape IV, February 2012. Cloudscape V, 27-28 February 2013 will keep the discussion of SIENA's calls to action alive building on the success of the SIENA initiative, and evaluating the calls for action emerging from the SIENA Roadmap a year on, assessing their current status globally and in Europe and with particular reference to the European Cloud Computing Strategy and its impact on the public and private sectors.

\section{References}

[1] SIENA, http://www.sienainitiative.eu/

[2] SIENA Roadmap on Distributed Computing Infrastructure for e-Science and Beyond in Europe, http://www.sienainitiative.eu/Pages/SelectedDocument.aspx?id documento=f63de70f7984-4dcd-9268-50eeadefb81a

[3] Cloudscape, http://www.cloudscapeseries.eu/

[4] The ESA/CERN proposal for a "science cloud", http://ur1.ca/9f2jf

[5] Digital Agenda Assembly 2011, Final Report, http://ur1.ca/9f2ki

[6] Workshop report 03: Interoperability and standards making it happen, http://ur1.ca/4dcxw

[7] Symposium Proceedings, OASIS International Cloud Symposium 2011, IEEE P2302

Standards for Intercloud Interoperability and Federation, ISO/IEC JTC1 SC38 Distributed

Applications Platforms and Services Working Group 3 Cloud Computing, and the Global Intercloud Technology Forum http://events.oasis-open.org/home/cloud/2011/proceedings

[8] US Government Cloud Computing Technology Roadmap, Release 1.0 (Draft) Volume 2:

Useful Information for Cloud Adopters,

http://www.nist.gov/itl/cloud/upload/SP 500293 volumeII.pdf

[9] NIST Inventory of Standards Relevant to Cloud Computing, http://ur1.ca/9f2t4

[10] US Government Cloud Computing Technology Roadmap, Release 1.0 (Draft) Volume 2:

Useful Information for Cloud Adopters, section 6, http://goo.gl/uBxFg

[11] Riding the wave: how Europe can gain from the rising tide of scientific data, http://ur1.ca/9f2wt 
[12] Justin Pirie, EU in danger of missing the point in cloud strategy debate, http://ur1.ca/9f311 [13] Cloud Computing: Public Consultation Report, http://ur1.ca/9f319

[14] SIENA Roadmap Annexes, www.sienainitiative.eu/SIENA Roadmap Annexes.aspx

[15] The Google contribution to the Public Consultation on Cloud Computing, http://ur1.ca/9f324

[16] Eurostars: Aim higher, http://www.eurostars-eureka.eu/

[17] European Commission: CORDIS: FP7: ICT: e-Infrastructure: Home, http://cordis.europa.eu/fp7/ict/e-infrastructure/

[18] Research Infrastructures - Research - European Commission, http://ec.europa.eu/research/infrastructures/index en.cfm

[19] https://documents.egi.eu/public/ShowDocument?docid=506

[20] Stephanie Parker, European initiatives strengthen cooperation on cloud computing VENUS-C, StratusLab and EDGI bring together cloud user communities and technology, http://ur1.ca/9f91v

[21] OpenNebula - The Open Source Solution for Data Center Virtualization, http://opennebula.org/

[22] OpenNebula - The Open Source Solution for Data Center Virtualization, Featured Users http://opennebula.org/users:users

[23] Capitalizing on e-infrastructure investments in Europe, Section5, http://compatibleone.org

[24] EGI-InSPIRE, http://www.egi.eu/projects/egi-inspire/

[25] The outcomes of the first 6 months activities, EGI Community Forum, 26-30 March 2012, Munich, Germany, https://wiki.egi.eu/wiki/Fedcloud-tf:FederatedCloudsTaskForce

[26] Open Grid Forum, http://www.gridforum.org

[27] Open Grid Forum Areas and Groups, http://www.ogf.org/gf/group info/view.php? group=dcifed-wg

[28] Notably P2301 and P2302 developed by the IEEE Standards Association, http://grouper.ieee.org/groups/2301/ and http://grouper.ieee.org/groups/2302/

[29] Asset Description Metadata Schema (ADMS)| Joinup,

https://joinup.ec.europa.eu/asset/adms/description

[30] OJEC (fficial Journal of the European Community) - welcome to OJEC, http://www.ojec.com/

[31] Welcome to the website of the e-Infrastructure Reflection Group, http://www.e-irg.eu/

[32] PRACE Research Infrastructure - The top level of the European HPC ecosystem,

http://www.prace-ri.eu 